\title{
2. SYNOPSIS
}

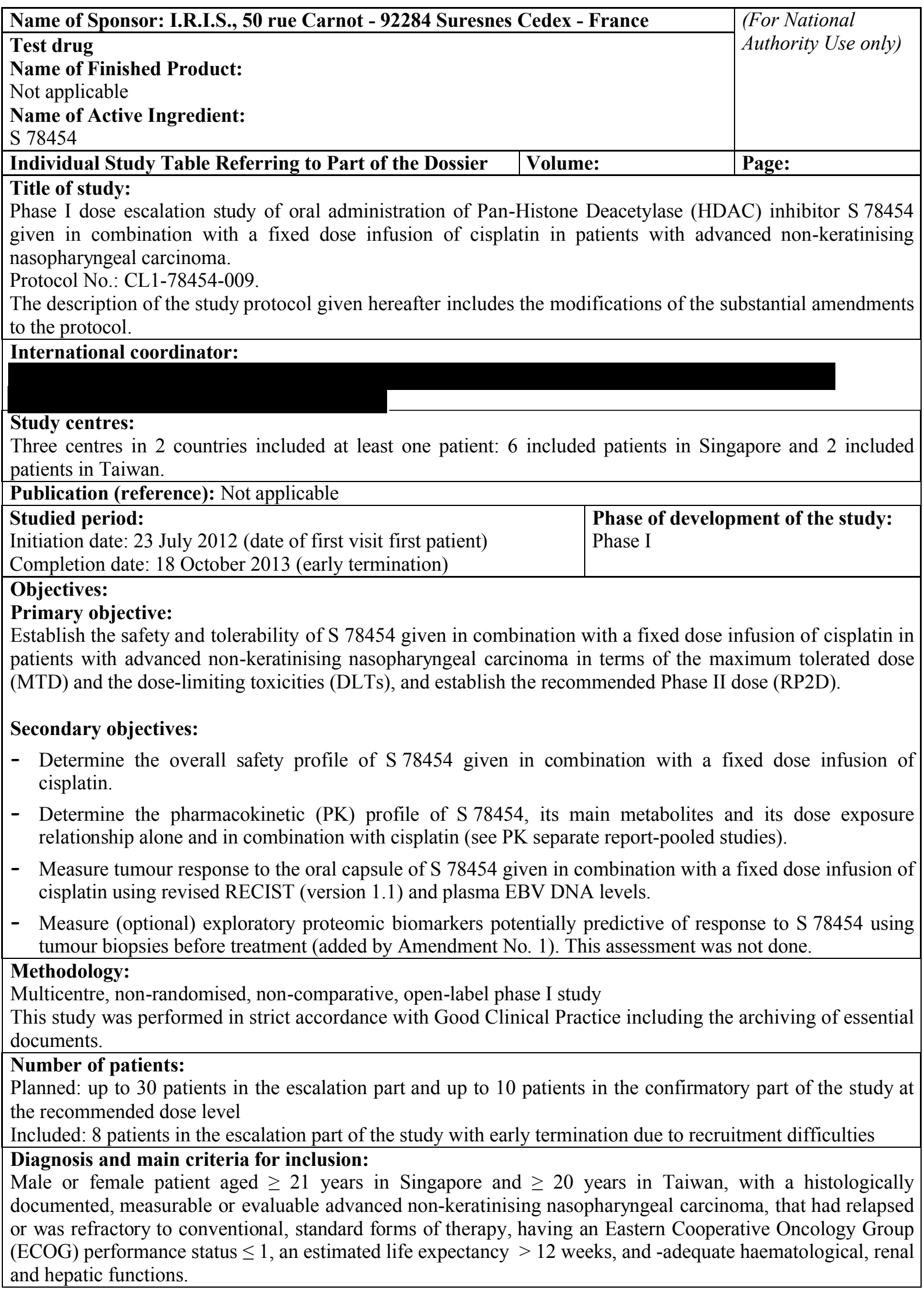




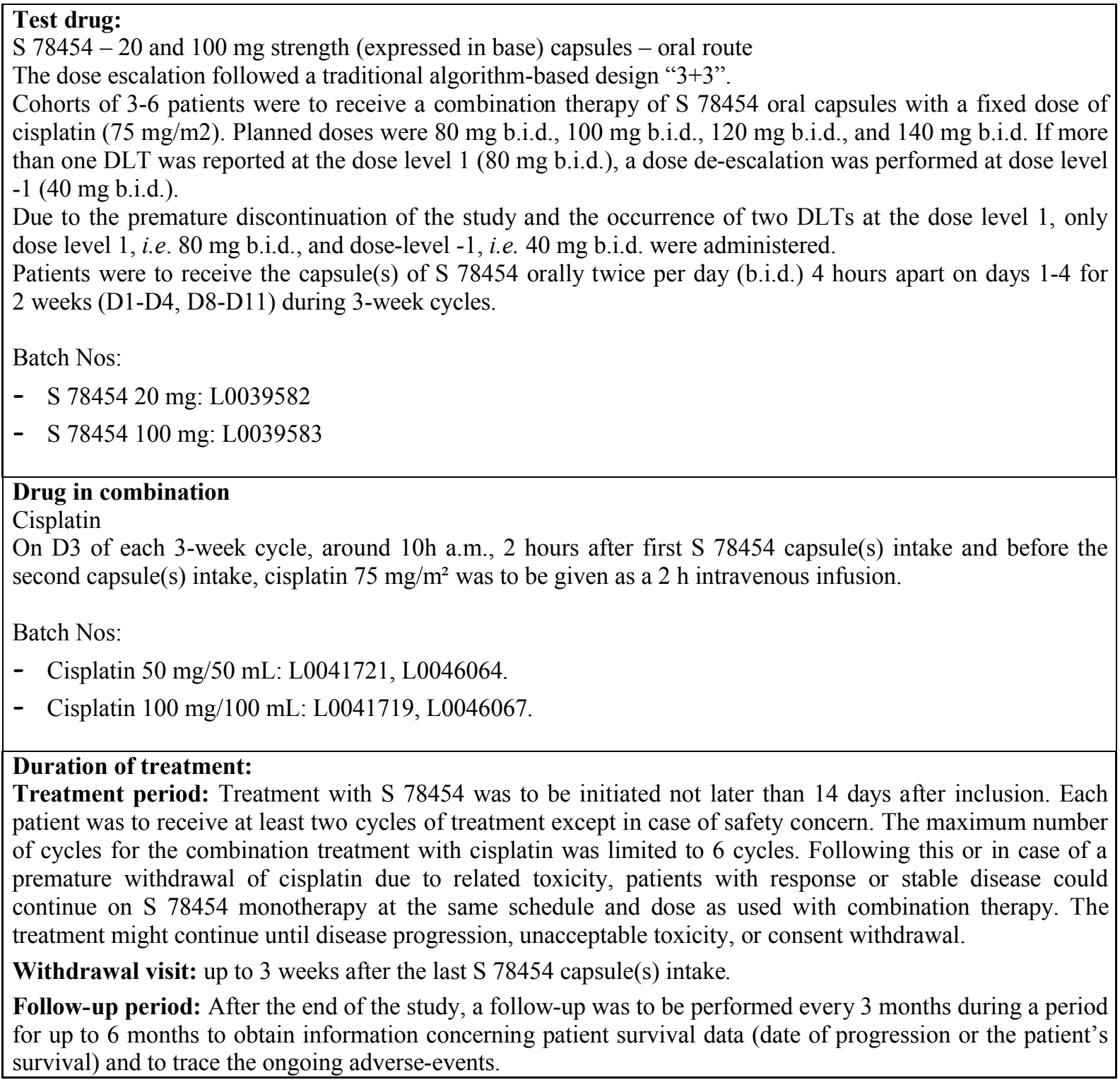




\section{Criteria for evaluation:}

Efficacy measurements:

Patients were evaluated for response, based on revised Response Evaluation Criteria in Solid Tumours (RECIST), at baseline within 4 weeks before the first drug administration and during day D18 to D21 of every 2 cycles beginning with cycle 2, and at the end of the study (this last tumour evaluation was at the investigator's discretion).

A response to the combination treatment was also evaluated by a plasma Epstein-Barr virus (EBV) DNA levels.

Safety measurements:

- Adverse events.

- Physical and neurological examination.

- Central QTcF interval measurement.

- Audiometric test.

- Laboratory examination.

Tolerance assessment was done according to the Common terminology criteria for adverse events (CTCAE) version v4.0.

Pharmacokinetic measurements:

Measurement of S 78454 and its metabolites were described in a separate protocol and results will be provided in a separate report.

Other measurements:

Optional exploratory proteomic biomarkers including HR23B using tumour biopsies collected before first treatment of S78454 (separate report)

\section{Statistical methods:}

Efficacy analysis (secondary objective): descriptive analysis of the best overall response.

Study outcome and safety analysis: Descriptive statistics.

Pharmacokinetic analysis: PK analyses will be described in a separate report (pooled studies).

Proteomic biomarkers analysis will be described in a separate report

\section{SUMMARY - CONCLUSIONS}

STUDY POPULATION AND OUTCOME

\begin{tabular}{lccc}
\hline & $\mathbf{8 0}$ mg b.i.d. & $\mathbf{4 0} \mathbf{~ m g ~ b . i . d . ~}$ & All \\
\hline Included & 4 & 4 & 8 \\
Withdrawn due to & 4 & 4 & 8 \\
- lost to follow-up & - & - & - \\
- adverse event & 3 & 1 & 4 \\
- progressive disease & - & 1 & 1 \\
- non-medical reason & 1 & 2 & 3 \\
- protocol deviation & - & - & - \\
Completed & - & - & - \\
Full Analysis Set (FAS) & 2 & 2 & 4 \\
Safety set & 4 & 4 & 8 \\
\hline
\end{tabular}

Overall, patients were $57.9 \pm 6.4$ years old and were mainly men $(62.5 \%)$.

A total of 8 patients were included in the study according to a traditional algorithm-based design " $3+3$ ". Four patients received S 78454 at Dose level 1, i.e. $80 \mathrm{mg}$ b.i.d., of whom two patients presented with DLT. Four patients were included at this dose level instead of 3 as one patient withdrew consent after one week of treatment and was replaced.

Four other patients received S 78454 at Dose level -1, i.e. $40 \mathrm{mg}$ b.i.d. of whom one patient experienced one DLT. After 3 included patients with 1 DLT, an extension to 6 patients was planned but stopped before the end due to recruitment difficulties.

As the study was stopped, the MTD could not be determined. 


\section{SUMMARY - CONCLUSIONS (Cont'd) \\ STUDY POPULATION AND OUTCOME (Cont'd)}

Two patients received $80 \mathrm{mg}$ b.i.d. during one cycle, one patient during 3 cycles and one patient, after one cycle at $80 \mathrm{mg}$ b.i.d. dose, experienced DLT and then received $40 \mathrm{mg}$ b.i.d. dose during the next two cycles.

In patients who started the $\mathrm{S} 78454$ treatment at $40 \mathrm{mg}$ b.i.d. dose, two patients were treated for one cycle, one patient for two cycles, and one patient for four cycles.

Cisplatin was administered at $75 \mathrm{mg} / \mathrm{m}^{2}$ dose except in one patient from the $\mathrm{S} 7845440 \mathrm{mg}$ b.i.d. group in whom the dose was reduced to $60 \mathrm{mg} / \mathrm{m}^{2}$ as requested in the protocol when creatinine clearance was under $60 \mathrm{~mL} / \mathrm{min}$.

All patients were withdrawn from the study, due to adverse events in four patients, non-medical reason in three patients, and progressive disease in one patient.

\section{EFFICACY RESULTS}

The response to treatment was available in 4 patients.

The best overall response was a partial response in 2 patients and a stable disease in 2 patients.

\section{SAFETY RESULTS}

\section{- Emergent adverse events}

\section{Overall summary of adverse events}

\section{ALL}

$(\mathbf{N}=\mathbf{8})$

Participants having reported

at least one emergent adverse event

Of which

- at least one S 78454 treatment-related emergent adverse event

- at least one S 78454 or cisplatin treatment-related emergent adverse event

- at least one cisplatin treatment-related emergent adverse event

Participants having experienced

at least one serious adverse event (including death)

at least one serious emergent event (including death)

at least one treatment-related* serious adverse event

Participants with treatment withdrawal

due to an adverse event

due to a serious adverse event

due a treatment-related adverse event

due a treatment-related* serious adverse event

Participants who died

$\begin{array}{lc}\text { n (\%) } & 8(100) \\ \text { n (\%) } & 2(25.0) \\ \text { n (\%) } & 7(87.5) \\ & \\ \text { n (\%) } & 4(50.0) \\ & \\ \text { n (\%) } & 3(37.5) \\ \text { n (\%) } & 3(37.5) \\ \text { n (\%) } & 3(37.5) \\ \text { n (\%) } & 4(50.0) \\ \text { n (\%) } & 2(25.0) \\ \text { n (\%) } & 4(50.0) \\ \text { n (\%) } & 2(25.0) \\ \text { n (\%) } & -\end{array}$

*relationship to $S 78454$ and cisplatin according to the investigator

During the study, all patients experienced at least one EAE. The most frequently affected system organs were: General disorders and administration site conditions (8 patients), Gastrointestinal disorders (7 patients), Blood and lymphatic system disorders (5 patients), and Metabolism and nutrition disorders (4 patients).

The most frequently reported ( $\geq 3$ patients) EAEs were fatigue (8 patients), thrombocytopenia (5 patients), nausea (4 patients), constipation ( 3 patients), diarrhoea ( 3 patients), anaemia ( 3 patients), and decreased appetite (3 patients).

Overall, 16/60 EAEs were severe (CTCAE grade $\geq 3$ ) of which the most frequent were thrombocytopenia in three patients ( 5 cases), anaemia in two patients, neutropenia in two patients, and fatigue in two patients. 


\section{SUMMARY - CONCLUSIONS (Cont'd)}

\section{SAFETY RESULTS (Cont'd)}

At Cycle 1, fatigue (grade 3) was considered as a DLT in two patients at dose level 1 ( $80 \mathrm{mg}$ b.i.d.) and renal impairment (grade 3) was a DLT in one patient at dose level -1 (40 mg b.i.d.). As the study was stopped prematurely, the MTD could not be determined.

Overall EAES were considered as related to S 78454 or cisplatin in 43/60 EAEs in 7/8 patients. Among these events, three cases of thrombocytopenia and one case of fatigue were considered by the investigator as related to $\mathrm{S} 78454$ only.

Five EAEs led to treatment withdrawal in four patients (2 SEAEs: anaemia and renal impairment, and 3 nonserious EAEs: 2 cases of fatigue and one case of nausea).

No patient died during the study. A total of 3 patients experienced 7 SEAEs (thrombocytopenia -2 cases; anaemia, constipation, pyrexia, chronic osteomyelitis, renal impairment, 1 case each), 5 being considered by the investigator as related to S 78454 and to cisplatin. They led to study treatment withdrawal in 2 cases (see above). All SEAEs recovered (chronic osteomyelitis, 2 cases of thrombocytopenia, constipation, pyrexia, renal impairment) or were recovering (anaemia).

In all, 5 patients experienced 8 thrombocytopeniae. Five cases were severe (CTCAE grade $\geq 3$ ), of which 2 cases were serious. All thrombocytopeniae recovered, four on treatment and four after stopping the treatment. None of them led to treatment withdrawal. Overall, the median values of nadir were lower in the $80 \mathrm{mg}$ b.i.d. group $(53.0 \mathrm{G} / \mathrm{L})$ than in the $40 \mathrm{mg}$ b.i.d. group $(105.5 \mathrm{G} / \mathrm{L})$, nevertheless no firm conclusion could be drawn on a dose effect due to the small sample sizes.

At baseline, all patients had a QTcF interval duration $\leq 450 \mathrm{~ms}$. During the study, one patient had an interval duration between 450 and $480 \mathrm{~ms}$ with a change from baseline $\leq 30 \mathrm{~ms}$. A change from baseline between 30 and $60 \mathrm{~ms}$ was observed in 3 other patients but with a QTcF interval duration $\leq 450 \mathrm{~ms}$.

\section{CONCLUSION}

In this Phase I study, two patients experienced a DLT at S $7845480 \mathrm{mg}$ b.i.d. dose, given in combination with cisplatin, and one patient presented with a DLT at $40 \mathrm{mg}$ b.i.d. dose. Due to the premature discontinuation of the study (because of difficulty in patient's recruitment), no MTD could be determined. The safety profile of $\mathbf{S} \mathbf{7 8 4 5 4}$ given in combination with cisplatin was in accordance with the expected safety profile of these products.

Date of the report: 07 October 2014

Version of the report: Final version 